\title{
Molecular definition of SARS-CoV-2 RBD mutations: receptor affinity versus neutralization of receptor interaction
}

\author{
Monique Vogel ${ }^{1}$, Gilles Augusto ${ }^{2}$, Xinyue Chang ${ }^{2}$, Xuelan $\mathrm{Liu}^{3}$, Daniel Speiser ${ }^{3}$, Mona \\ Mohsen $^{2}$, and Martin Bachmann ${ }^{3}$ \\ ${ }^{1}$ Inselspital University Hospital Berne Institute of Immunology \\ ${ }^{2}$ University of Bern \\ ${ }^{3}$ Inselspital University Hospital Bern
}

April 21, 2021

\begin{abstract}
Background: Several new variants of SARS-CoV-2 have emerged since fall 2020 which have multiple mutations in the receptor binding domain (RBD) of the spike protein. It is unclear which mutations affect receptor affinity versus immune recognition. Methods: We produced RBD with single mutations (E484K, K417N or N501Y) or with all three mutations combined and tested their binding to ACE2 by biolayer interferometry (BLI). The ability of convalescent sera to recognize RBDs and block their interaction with ACE2 was tested as well. Results: We demonstrated that single mutation N501Y increased binding affinity to ACE2 but did not significantly affect its recognition by convalescent sera. In contrast, single mutation E484K had almost no impact on the binding kinetics, but essentially abolished recognition of RBD by convalescent sera. Interestingly, combining mutations E484K, K417N and N501Y resulted in a RBD with both features: enhanced receptor binding and abolished immune recognition. Conclusion: Our data demonstrate that single mutations either affect receptor affinity or immune recognition while triple mutant RBDs combine both features.
\end{abstract}

Molecular definition of SARS-CoV-2 RBD mutations: receptor affinity versus neutralization of receptor interaction

Monique Vogel ${ }^{1,2 \&}$, Gilles Sousa Augusto ${ }^{1,2 \&}$, Xinyue Chang ${ }^{1,2}$, Xuelan Liu ${ }^{1,3}$, Daniel Speiser ${ }^{1,2}$, Mona O. Mohsen $^{1,2}$ and Martin F. Bachmann ${ }^{1,2,3}$ *

\section{Affiliations}

${ }^{1}$ Department of Immunology, University clinic of Rheumatology and Immunology, Inselspital, Bern, Switzerland

${ }^{2}$ Department of BioMedical Research, University of Bern, Bern, Switzerland

${ }^{3}$ International Immunology Centre, Anhui Agricultural University, Hefei, China, Dermatology,

${ }^{4}$ Nuffield Department of Medicine, Centre for Cellular and Molecular Physiology (CCMP),

The Jenner Institute, University of Oxford, Oxford, UK

\& equal contribution

\section{Address for Correspondence:}

Martin Bachmann, University Clinic of Rheumatology and Immunology, University Hospital Bern, Salihaus 2, CH-3010 Bern, Switzerland. E-mail: martin.bachmann@dbmr.unibe.ch. 


\section{Acknowledgement}

We thank Marianne Zwicker for production of wild type and mutant RBDs. We thank PD Dr. Alexander Eggel and Dr. Daniel Brigger for providing biotinylated and non-biotinylated ACE2-mFc.

\section{Funding}

We thank Saiba AG, the Swiss National Science Foundation (SNF grant 31003A_185114) and the International Immunology Centre, Anhui Agricultural University, Hefei, China for the financial support.

\section{Conflict of Interests}

M. F. Bachmann is a board member of Saiba AG, involved in the development of RBD-CuMV a vaccine against COVID-19. All other authors declare no conflict of interest

\section{Author contributions}

M.V., G.S.A., X.C. designed, performed and interpreted most experiments; X.L. M.M. provided serum sample; M.V., G.S.A., D.S. wrote the manuscript, M.F.B. designed experiments and wrote the manuscript.

\section{Abstract}

Background : Several new variants of SARS-CoV-2 have emerged since fall 2020 which have multiple mutations in the receptor binding domain (RBD) of the spike protein. It is unclear which mutations affect receptor affinity versus immune recognition.

Methods: We produced RBD with single mutations (E484K, K417N or N501Y) or with all three mutations combined and tested their binding to ACE2 by biolayer interferometry (BLI). The ability of convalescent sera to recognize RBDs and block their interaction with ACE2 was tested as well.

Results: We demonstrated that single mutation N501Y increased binding affinity to ACE2 but did not significantly affect its recognition by convalescent sera. In contrast, single mutation E484K had almost no impact on the binding kinetics, but essentially abolished recognition of RBD by convalescent sera. Interestingly, combining mutations E484K, K417N and N501Y resulted in a RBD with both features: enhanced receptor binding and abolished immune recognition.

Conclusions: Our data demonstrate that single mutations either affect receptor affinity or immune recognition while triple mutant RBDs combine both features.

\section{Keywords}

SARS-CoV-2, RBD, affinity, neutralization, ACE2, biolayer interferometry

\section{Introduction}

SARS-CoV-2 has caused a pandemic that is characterized in many countries by several waves of infection ${ }^{1}$

2. While the origin of these infection waves may differ in different regions of the world, the latest increase seen in numbers of infected individual is apparently caused by the occurrence of mutated viral strains ${ }^{3}$. The most prominent mutated strains are following variants: B.1.1.7. (N501Y, D614G), P.1 (K417N/T, E484K, N501Y) and B.1.351 (K417N, E484K, N501Y, D614G) ${ }^{4}$, which show mutations in the receptor binding domain (RBD) and receptor binding motif (RBM) of the spike (S) glycoprotein. $\mathrm{RBD}$ and in particular RBM are responsible for interaction with the cellular receptor ACE2 and are the primary target of neutralizing antibodies ${ }^{5}$ (Fig.1). Mutant viruses may spread more efficiently because they show increased affinity for the receptor or because they escape neutralizing antibody responses. The importance of receptor affinity has been illustrated by SARS-CoV-1, which showed a 4-fold lower affinity for ACE2 and also was much less contagious and showed strongly reduced transmission compared to SARS-CoV-2 ${ }^{6}$.

Viruses that escape neutralization are typically called serotypes and usually may only occur when a large proportion of individuals show antibody-based immunity against the original strain and further spread may only be possible by escape of neutralizing antibody responses ${ }^{7}$. For SARS-CoV-2, this is certainly not the 
case on a global scale but certain regions of the world may show infections rates that are compatible with serotype formation.

Here we assessed the molecular basis for antibody escape and how the RBD mutations present in two variants of concern (B.1.1.7 and P.1) influence the affinity to the receptor.

\section{Material and Methods}

\section{Protein expression and purification}

The SARS-CoV-2 receptor-binding domain $\left(\mathrm{RBD}_{\mathrm{WT}}\right)$ and the RBD mutant $\left(\mathrm{RBD}_{\mathrm{K} 417 \mathrm{~N}}, \mathrm{RBD}_{\mathrm{N} 501 \mathrm{Y}}\right.$, $\mathrm{RBD}_{\mathrm{K} 417 \mathrm{~N}}, \mathrm{RBD}_{\mathrm{TRIP}}$ ) were expressed using Expi293F cells (Invitrogen, ThermoFisher Scientific, MA, USA).

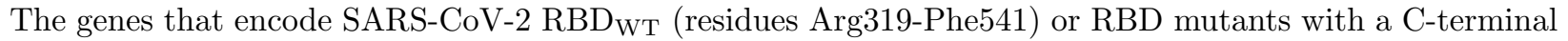
6-His-tag was inserted into pTwist CMV BetaGlobin WPRE Neo vector (Twist Bioscience, San Francisco, USA). The construct plasmids were transfected into Expi293F cells using ExpiFectatmine 293 transfection kit (Gibco, ThermoFisher Scientific, MA, USA). The supernatant of cell culture containing the secreted RBDs was purified using His-Trap HP column (GE Healthcare, USA). Collected RBD ${ }_{\text {WT }}$ or RBD mutated proteins were equilibrated in PBS and kept at -20@C. ACE2-mFc was purchased from Sino Biological (Eschborn, Germany). Biotinylated and non-biotinylated soluble human ACE2 fused to mouse IgG2a Fc proteins were kindly provided by PD Dr. Alexander Eggel (University Clinic of Rheumatology and Immunology, Inselspital, Bern, Switzerland) who received the plasmid from Prof. Peter Kim (Stanford University, CA, USA).

\section{Human sera}

Human sera were obtained from 11 COVID-19 convalescent patients which were recruited the University Hospital of Bern, Bern, Switzerland ${ }^{13}$. All patients provided written informed consent before participation in the studies.

\section{ELISA Assay}

Corning half area 96-well plates were coated with $1 \mu \mathrm{g} / \mathrm{ml} \mathrm{RBD}_{\mathrm{WT}}$ or mutated RBDs in PBS overnight at $4^{\circ} \mathrm{C}$ and then blocked with PBS $/ 0.15 \%$ casein. Convalescent human sera were added, serially diluted 1:3 and incubated on plates for one hour at room temperature. Bound IgG antibodies were detected with goat anti-human IgG-POX antibody (Nordic MUbio, Susteren, The Netherlands). ELISA was developed with tetramethylbenzidine (TMB), stopped by adding equal $1 \mathrm{M} \mathrm{H}_{2} \mathrm{SO}_{4}$ solution, and read at $\mathrm{OD}_{450 \mathrm{~nm}}$.

\section{RBDwt and RBDmut kinetics by Bio-Layer Interferometry (BLI)}

The analysis of binding kinetics of $\mathrm{RBD}_{\mathrm{WT}}$ and $\mathrm{RBD}_{\mathrm{TRIP}}$ to $\mathrm{ACE} 2-\mathrm{mFc}$ was performed by Bio-Layer Interferometry (BLI) using an Octet RED96e (Fortebio) instrument. High precision Streptavidin (SAX, ForteBio, Fremont, CA, USA) biosensors were saturated with $7.5 \mu \mathrm{g} / \mathrm{ml}$ biotinylated ACE2-mFc in BLI assay buffer (PBS, 0.1\% BSA, $0.02 \%$ Tween 20) for 10 min. $\mathrm{RBD}_{\mathrm{WT}}$ and $\mathrm{RBD}_{\mathrm{TRIP}}$ were prepared as twofold serial dilution (typically 50, 25, 12.5, 6.25 and $3.125 \mathrm{nM}$ ) in BLI assay buffer plus buffer blanks. Kinetic values were calculated by ForteBio data analysis software using a 1:1 binding model.

\section{BLI-based competitive assay}

The ability of the sera of the COVID convalescent patient to compete with ACE2 for binding to RBD WT $_{\text {and }}$ $\mathrm{RBD}_{\text {TRIP }}$ was tested in a sandwich format assay on the Octet RED96e (Fortebio). Anti-penta-His (HIS1K) biosensors were loaded for 10 min with $\mathrm{RBD}_{\mathrm{WT}}$ and $\mathrm{RBD}_{\text {TRIP }}$ at a concentration of $7.5 \mu \mathrm{g} / \mathrm{ml}$ in BLI assay buffer followed by addition of samples (diluted 1:20 in BLI assay buffer) from convalescent human sera. To assess whether the sera can inhibit the binding of ACE2 to RBD $\mathrm{WT}_{\text {T }}$ and $\mathrm{RBD}_{\mathrm{TRIP}}$, ACE2-mFc (50 nM) was added to biosensor. For control two additional sensors with BLI buffer were used, one for baseline and one without serum sample to determine binding of ACE2-mFc alone. The results are expressed of single individual. The response data were normalized using ForteBio data analysis software version1.2.0.1.55.

Data and statistical analysis 
All statistical tests were performed using GraphPad PRISM 8.0 (GraphPad Software, Inc.). ELISA data in graphs are displayed as enpoint titres measured at a cut off 0.15 OD $450 \mathrm{~nm}$. Comparison between $\mathrm{RBD}_{\mathrm{WT}}$ and mutated RBDs were analyzed by two-ways Anova test for ELISA and paired two-tailed Student's t-test for BLI assay. $\alpha=0.05$ and statistical significance are displayed as p[?]0.05 $\left(^{*}\right), \mathrm{p}[?] 0.01\left(^{* *}\right), \mathrm{p}[?] 0.005\left(^{* * *}\right)$, $\mathrm{p}[?] 0.001(* * * *)$.

\section{Results}

\section{Structural model and binding kinetics of SARS-CoV-2 RBD variants to ACE2}

To address the questions of antibody binding strength and competition mechanistically, we have expressed the RBD of the isolate P.1 exhibiting RBD mutations (K417N, E484K and N501Y) (RBD Trip), two of which are located in the RBM (E484K, N501Y) (shown in Fig. 1A) ${ }^{8}$. In addition, we generated each of the respective single mutants $\left(\mathrm{RBD}_{\mathrm{K} 417 \mathrm{~N}}, \mathrm{RBD}_{\mathrm{E} 484 \mathrm{~K}}\right.$, and $\left.\mathrm{RBD} \mathrm{D}_{\mathrm{N} 501 \mathrm{Y}}\right)$. All $\mathrm{RBDs}$ were purified to homogeneity and the affinity to recombinant ACE2 was determined by Biolayer Interferometry using Octet technology 9. The BLI assays showed that the affinity of ACE2 for $\mathrm{RBD}_{\mathrm{TRIP}}$ (shown in Fig. $2 \mathrm{~B}, 2 \mathrm{~F}$, Table $1, \mathrm{~K}_{\mathrm{D}}=$ $10.3 \mathrm{nM}$ ) was about twice as high as for $\mathrm{RBD}_{\mathrm{WT}}$ (shown in Fig. $2 \mathrm{~A}, 2 \mathrm{~F}$, Table $1, \mathrm{~K}_{\mathrm{D}}=20.5 \mathrm{nM}$ ). Having in mind that the affinity of the of SARS-CoV-2 for ACE2 is only 4-fold higher compared to SARS-CoV-1, this factor of 2 is expected to be biologically significant and most likely reflects enhanced infectivity. In contrast, the mutation at position 484 in $\mathrm{RBD}_{484}$ did not affect receptor affinity (shown in Fig. 2D, F). For comparison, the dissociation constant observed for $\mathrm{RBD}_{\mathrm{N} 501 \mathrm{Y}}$ was 3 -fold lower $\left(\mathrm{K}_{\mathrm{D}}=6.2 \mathrm{nM}\right.$, shown in Fig. $2 \mathrm{C}, 2 \mathrm{~F}$, Table 1). Interestingly, the mutation at position 417 in $\mathrm{RBD}_{417}$ resulted in completely altered binding properties (shown in Fig. $2 \mathrm{E}$ ). $\mathrm{RBD}_{\mathrm{K} 417 \mathrm{~N}}$ showed much lower association rates and plateau levels a non-monovalent pattern of dissociation rates (shown in Fig. $2 \mathrm{E}$, Table $1 \mathrm{~K}_{\mathrm{D}}$ could not be determined in a meaningful way). Presence of aggregates was not responsible for this effect, as purification by size exclusion immediately before measurements did not alter the binding kinetics observed (data not shown). However, since this mutation does not occur on its own in viral strains, we did not further investigate this effect.

\section{Reduced ability of convalescent sera to recognize RDB variants}

To determine whether $\mathrm{RBD}_{\mathrm{WT}}$-specific immune sera might have a reduced ability to bind to mutated RBDs we performed ELISA and Biolayer Interferometry using sera from convalescent patients (shown in Fig. 3). As expected $\mathrm{RBD}_{\mathrm{WT}}$ was well recognized by convalescent sera in ELISA experiments. In contrast, $\mathrm{RBD}_{\mathrm{K} 417 \mathrm{~N}}$ and $\mathrm{RBD}_{\mathrm{N} 501 \mathrm{Y}}$ were recognized in a slightly impaired fashion (shown in Fig. 3A). In marked contrast, mutation at position 484 essentially abolished recognition of both $\mathrm{RBD}_{\mathrm{E} 484 \mathrm{~K}}$ and $\mathrm{RBD}_{\mathrm{TRIP}}$. Corresponding results were obtained using Biolayer Interferometry (shown in Fig. 3B). RBD-specific neutralizing antibodies typically block interaction of RBD with the viral receptor ACE2. We therefore assessed whether reduced binding of convalescent sera to $\mathrm{RBD}_{\mathrm{TRIP}}$ was paralleled by reduced ability of these antibodies to block binding of ACE2 to the triple mutant (shown in Fig. 3C). These experiments demonstrate that human convalescent sera essentially failed to block binding of ACE2 to RBD TRIP, explaining why SARS-CoV-2induced antibodies largely fail to neutralize the triple mutant variants.

\section{Discussion}

The newly emerging mutant RBDs may affect the affinity for the viral receptor. In addition, such mutations at the virus-receptor interaction face may alter the ability of RBD-specific antibodies - induced by previous infection - to neutralize the mutant viruses. A previous study showed that serum neutralization is not compromised by N501Y (found in the strain B.1.1.7) ${ }^{10}$. In contrast, E484K (found in B.1.1.7 and in P.1 strains) was associated with reduced neutralization ${ }^{11,12}$. Interestingly, studies applying in vitropressure produced similar mutations as those that occurred naturally ${ }^{13}$. When we investigated whether distinct mutations may affect receptor affinity we found that N501Y mutation enhanced affinity for the viral receptor ACE2 both as a single mutation as well as in the triple mutation, while E484K mutation alone did not affect the interaction with ACE2 .

In addition, such mutations at the virus-receptor interaction interface may alter the ability of RBD-specific 
antibodies to recognize and neutralize the mutant variants. A previous study showed that serum neutralization is not compromised by N501Y (also found in the strain B.1.1.7) ${ }^{10}{ }^{14}$. In contrast, E484K (found B.1.1.7 and in P.1 strains) was associated with reduced neutralization ${ }^{11,12}$. Interestingly, studies applying in vitro pressure produced similar mutations as those that occurred naturally ${ }^{13}$. In this study we showed that convalescent sera has reduced ability to recognize $\mathrm{RDB}_{\text {TRIP }}$ variants explaining why the mutant SARS-CoV-2 strain P.1 is more infectious ${ }^{15}$ and less susceptible to neutralization by antibodies induced with $\mathrm{RBD}_{\mathrm{WT}}$.

In summary, our data demonstrate that distinct mutations may affect receptor affinity which likely affects viral infectivity versus recognition by convalescent sera which likely affects neutralization. These observations may shed light on the potential origin of the viral mutants. The variant with the mutation N501Y shows enhanced affinity but almost normal recognition by convalescent antibodies. This indicates that this variant spread largely by increased infectivity while recognition by antibodies of previously infected individuals was less relevant, a phenotype consistent with the epidemiology in the UK, where overall infection rates remain relatively low, rendering the previously infected individuals a relatively unimportant source of viral spread ${ }^{16}$. In contrast, the triple mutant variant shows enhanced infectivity and escape from antibody-recognition. It may therefore not be a coincidence that this variant originated in Manaus, a region in Brazil previously seen to have seroprevalence of $>80 \%$, forcing the virus to escape immunity for further spreading ${ }^{17}$.

\section{References}

1. Choudhary S, Sreenivasulu K, Mitra P, Misra S, Sharma P. Role of Genetic Variants and Gene Expression in the Susceptibility and Severity of COVID-19. Ann Lab Med. 2021;41(2):129-138.

2. Bontempi E. The europe second wave of COVID-19 infection and the Italy "strange" situation. Environ Res. 2021;193:110476.

3. Bakhshandeh B, Sorboni SG, Javanmard AR, et al. Variants in ACE2; potential influences on virus infection and COVID-19 severity.Infect Genet Evol. 2021;90:104773.

4. Zhao S, Lou J, Cao L, et al. Quantifying the transmission advantage associated with N501Y substitution of SARS-CoV-2 in the United Kingdom: An early data-driven analysis. J Travel Med. 2021.

5. Yi C, Sun X, Ye J, et al. Key residues of the receptor binding motif in the spike protein of SARS-CoV-2 that interact with ACE2 and neutralizing antibodies. Cell Mol Immunol. 2020;17(6):621-630.

6. Huang Y, Yang C, Xu XF, Xu W, Liu SW. Structural and functional properties of SARS-CoV-2 spike protein: potential antivirus drug development for COVID-19. Acta Pharmacol Sin.2020;41(9):1141-1149.

7. Bachmann MF, Zinkernagel RM. The influence of virus structure on antibody responses and virus serotype formation. Immunol Today.1996;17(12):553-558.

8. Faria NR, Mellan TA, Whittaker C, et al. Genomics and epidemiology of a novel SARS-CoV-2 lineage in Manaus, Brazil. medRxiv. 2021.

9. Petersen RL. Strategies Using Bio-Layer Interferometry Biosensor Technology for Vaccine Research and Development. Biosensors (Basel). 2017;7(4).

10. Xie X, Liu Y, Liu J, et al. Neutralization of SARS-CoV-2 spike 69/70 deletion, E484K and N501Y variants by BNT162b2 vaccine-elicited sera.Nat Med. 2021.

11. Wibmer CK, Ayres F, Hermanus T, et al. SARS-CoV-2 501Y.V2 escapes neutralization by South African COVID-19 donor plasma. bioRxiv.2021.

12. Greaney AJ, Starr TN, Gilchuk P, et al. Complete Mapping of Mutations to the SARS-CoV-2 Spike Receptor-Binding Domain that Escape Antibody Recognition. Cell Host Microbe. 2021;29(1):44-57 e49.

13. Andreano E, Piccini G, Licastro D, et al. SARS-CoV-2 escape in vitro from a highly neutralizing COVID-19 convalescent plasma. bioRxiv.2020. 
14. Rathnasinghe R, Jangra S, Cupic A, et al. The N501Y mutation in SARS-CoV-2 spike leads to morbidity in obese and aged mice and is neutralized by convalescent and post-vaccination human sera.medRxiv. 2021.

15. Corum J, Zimmer C. Coronavirus variants and mutations. The New York Times. March 22, 2021, 2021.

16. News B. Coronavirus infection levels continue to drop in the UK. In. 5 March ed2021.

17. Candido DS, Claro IM, de Jesus JG, et al. Evolution and epidemic spread of SARS-CoV-2 in Brazil. Science.2020;369(6508):1255-1260.

Table 1 Kinetic parameters of $\mathrm{RBD}_{\mathrm{WT}}$ and mutated RBDs calculated by BLI

\begin{tabular}{llll}
\hline Analyte & $\mathbf{K}_{\mathbf{D}}[\mathbf{M}]$ & $\mathbf{k}_{\text {on }}\left[\mathbf{M}^{-\mathbf{1}} \mathbf{s}^{-\mathbf{1}}\right]$ & $\mathbf{k}_{\text {off }}\left[\mathbf{s}^{\mathbf{- 1}}\right]$ \\
\hline RBD $_{\mathrm{WT}}$ & $20.5 \times 10^{-9}$ & $1.34 \times 10^{5}$ & $2.91 \times 10^{-3}$ \\
$\mathrm{RBD}_{\mathrm{TRIP}}$ & $10.3 \times 10^{-9}$ & $1.69 \times 10^{5}$ & $1.75 \times 10^{-3}$ \\
$\mathrm{RBD}_{\mathrm{N} 501 \mathrm{Y}}$ & $6.2 \times 10^{-9}$ & $1.69 \times 10^{5}$ & $1.85 \times 10^{-3}$ \\
$\mathrm{RBD}_{\mathrm{E} 484 \mathrm{~K}}$ & $19.7 \times 10^{-9}$ & $1.64 \times 10^{5}$ & $3.24 \times 10^{-3}$ \\
$\mathrm{RBD}_{\mathrm{K} 41 \mathrm{~N}}$ & $\mathrm{ND}^{*}$ & $\mathrm{ND}^{*}$ & $\mathrm{ND}^{*}$ \\
\hline
\end{tabular}

$* \mathrm{ND}=$ not determined

\section{Figure Legends}

Fig. 1 Key mutations of variants B.1.351 and P1 fall on the interface between the RBD and ACE2. (left) S monomer (purple ribbon) bound to ACE2 ectodomain (orange surface). In detail, the positions of residues K417, E484, and N501 (blue sticks) are highlighted. Mutations E484K and N501K occur on the RBM segments (dark purple ribbon), while K417N occurs on helix $\alpha 4$ of RBD. From PDB files 6ACG and 6M0J.

Fig. 2 Binding kinetics of $\mathrm{RBD}_{\mathrm{WT}}(\mathrm{A}), \mathrm{RBD}_{\mathrm{TRIP}}(\mathrm{B}), \mathrm{RBD}_{\mathrm{N} 501 \mathrm{Y}}(\mathrm{C}), \mathrm{RBD}_{\mathrm{E} 484 \mathrm{~K}}(\mathrm{D})$, and $\mathrm{RBD}_{\mathrm{K} 417 \mathrm{~N}}(\mathrm{E})$ to hACE2. In all assays, both association and dissociation were performed in $300 \mathrm{~s}$. The resulting $\mathrm{k}_{\mathrm{on}}$ and $\mathrm{k}_{\mathrm{off}}$ values from each condition are compared in panel $(\mathrm{F})$.

Fig. 3 Sera of COVID-19 convalescent patients bind less to $\mathrm{RBD}_{\mathrm{WT}}$ and mutated RBDs $\left(\mathrm{RBD}_{\mathrm{TRIP}}\right.$, $\mathrm{RBD}_{\mathrm{N} 501 \mathrm{Y}}, \mathrm{RBD}_{\mathrm{E} 484 \mathrm{~K}}$, and $\left.\mathrm{RBD}_{\mathrm{K} 417 \mathrm{~N}}\right)$. A) Binding of sera from 12 COVID-19 convalescent patients and one COVID negative individual to $\mathrm{RBD}_{\mathrm{WT}}$ and mutated RBDs was determined by ELISA. Endpoint titres (EPT) (dilution factor) individuals are shown as dots. Direct binding of sera to $\mathrm{RBD}_{\mathrm{WT}}$ and mutated RBDs (B) and competitive inhibition of ACE2-mFc interaction to $\mathrm{RBD}_{\mathrm{WT}}$ and mutated $\mathrm{RBD}(\mathrm{C})$ were assessed by BLI. The same sera (dilution 1:20) for each individual was usual. p[?]0.05 $\left(^{*}\right), \mathrm{p}[?] 0.01\left(^{* *}\right), \mathrm{p}[?] 0.005\left(^{* * *}\right)$, $\mathrm{p}[?] 0.001(* * * *)$.

\section{Figure 1}




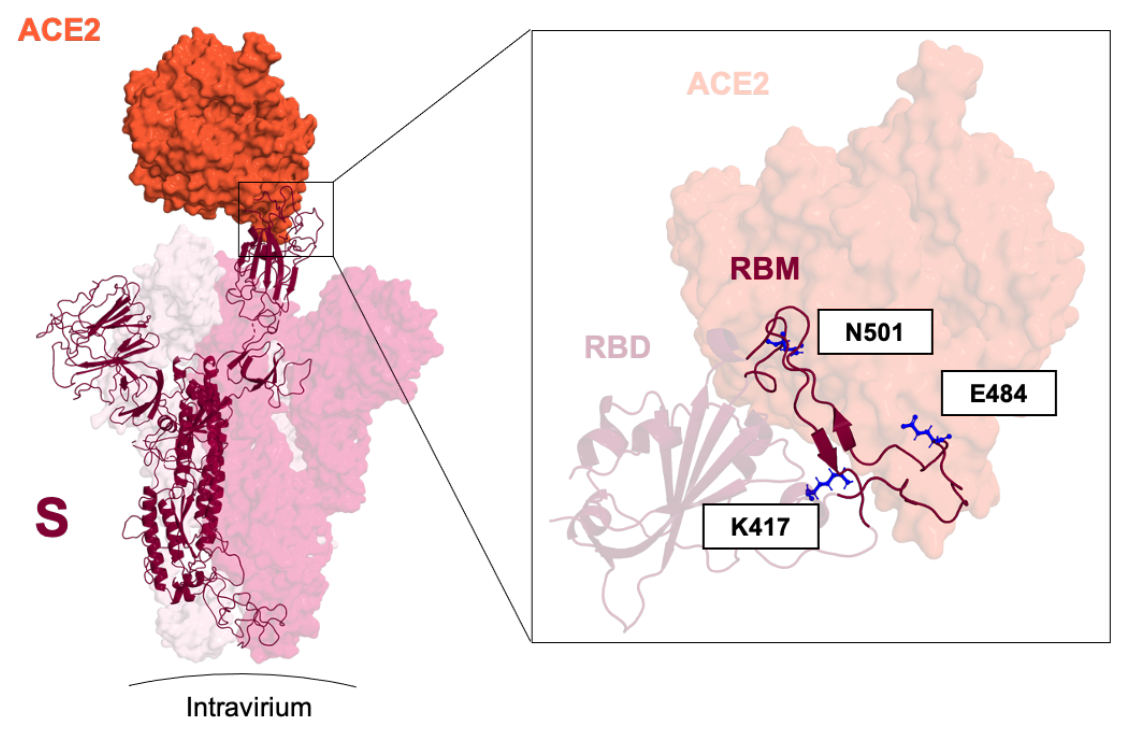

Figure 2

A.

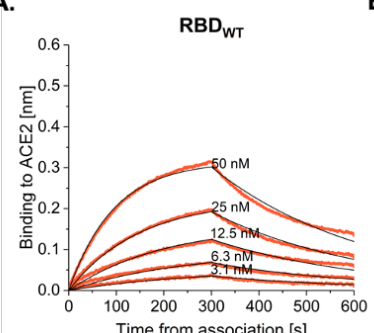

D.

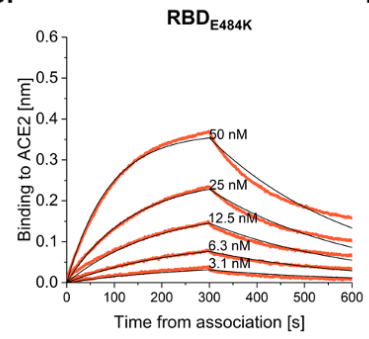

B.

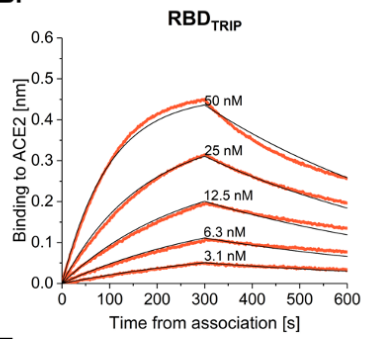

E.

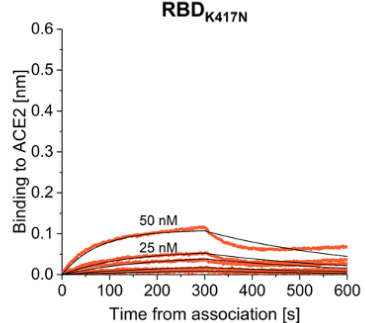

c.

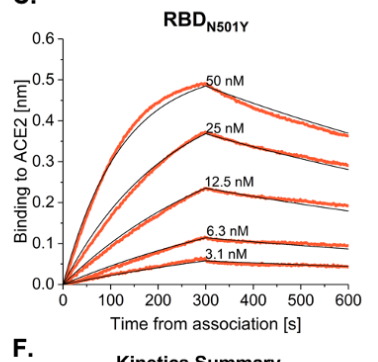

F.

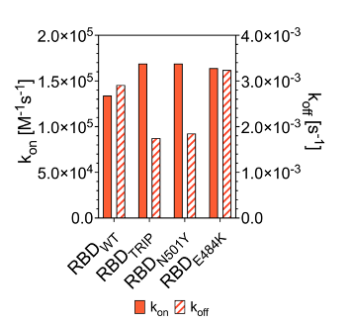

Figure 3 
B.

A.

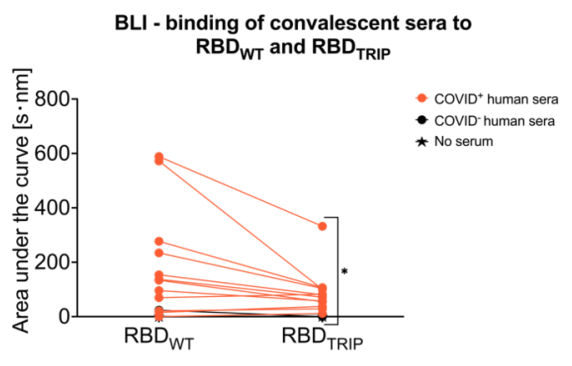

c.

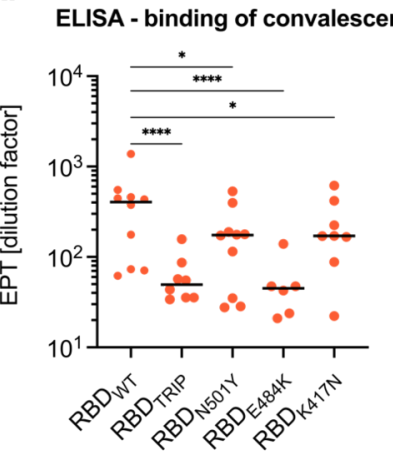

. BLI - inhibition of ACE2 binding to $R B D_{W T}$ and $R B D_{T R I P}$ by

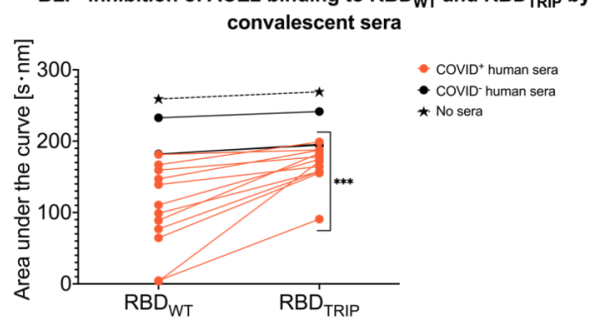

